\title{
Sintomas de alergia em idosos atendidos em um ambulatório de geriatria
}

\author{
Symptoms of allergy in elderly attended by a geriatric outpatient clinic
}

\author{
Lilian Dias dos Santos Alves', Zamir Calamita ${ }^{2}$
}

${ }^{1}$ Enfermeira, Mestre pelo Programa Saúde e Envelhecimento da Faculdade de Medicina de Marília (FAMEMA), Marília, SP; ${ }^{2}$ Médico, Professor e Chefe da Disciplina de Alergia e Imunologia da FAMEMA, Marília, SP.

\section{RESUMO}

Objetivos: Descrever as características epidemiológicas de manifestações alérgicas observadas nos idosos atendidos em um ambulatório de geriatria.

Métodos: Foi realizado um estudo transversal com pacientes de mais de 60 anos de idade, atendidos no Ambulatório de Geriatria da Faculdade de Medicina de Marília, estado de São Paulo, em condições de responder a uma entrevista ou com acompanhantes capacitados para respondê-la.

Resultados: Foram entrevistados 106 pacientes. Dentre os 106 pacientes avaliados, 56 (52,8\%) relataram alergia respiratória, 47 (44,3\%) possível manifestação alérgica de pele e $14(13,2 \%)$ referiram alergia a medicamentos. Os principais medicamentos responsabilizados pelos pacientes como causadores de reações alérgicas foram os antibióticos $(42,8 \%)$ e os anti-inflamatórios não esteroides $(28,6 \%)$, enquanto os outros $28,6 \%$ foram atribuídos a outros tipos de medicamentos.

Conclusões: Os idosos incluídos no estudo apresentaram uma relevante prevalência de sintomas alérgicos.

DESCRITORES: ALERGIA; HIPERSENSIBILIDADE; IDOSO.

\section{ABSTRACT}

Aims: To describe the epidemiological characteristics of allergic manifestations observed in elderly attended in an outpatient clinic of geriatrics. Methods: A cross sectional study was conducted with patients over 60 years of age, attended in the Outpatient Clinics of Geriatrics of the Faculty of Medicine of Marilia in Sao Paulo state, who were able to respond to an interview or were accompanied by caregivers able to do it. Results: A total of 106 patients were interviewed. Among the 106 patients evaluated, $56(52.8 \%)$ reported respiratory allergy, 47 (44.3\%) possible allergic skin manifestation, and $14(13.2 \%)$ reported drug allergies. The main medicines perceived by patients as drugs that caused allergic reactions were antibiotics $(42.8 \%)$ and non-steroidal anti-inflammatory $(28.6 \%)$, while the other $28.6 \%$ of reactions were attributed to other types of drugs.

Conclusions: The elderly included in the study had a relevant prevalence of allergic symptoms.

KEY WORDS: ALLERGY; HYPERSENSITIVITY; AGED. 


\section{INTRODUÇÃO}

O aumento progressivo da população idosa e a existência de poucos estudos abordando a doença alérgica no idoso fazem deste assunto um campo novo e interessante para pesquisa. $\mathrm{O}$ envelhecimento humano leva a uma diminuição da proliferação e funcionalidade celular, o que provoca um retardamento no funcionamento do organismo, gerando um impacto significativo nos aspectos biopsicossocial, espiritual e na multidimensionalidade da saúde na vida do indivíduo. ${ }^{1}$ No que se refere ao sistema imunológico, o idoso apresenta uma maior susceptibilidade ao desenvolvimento de doenças como câncer e infecções. Quanto aos aspectos da resposta alérgica, assim como sua expressão no indivíduo idoso, poucos estudos estão

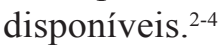

O processo alérgico é desencadeado por antígenos, substâncias identificadas pelo sistema imunológico como estranhas ao organismo. Um dos mecanismos mais relacionados com o desencadeamento das alergias ocorre pela produção de anticorpos do tipo imunoglobulina $\mathrm{E}$, que vão reagir contra os antígenos nos mastócitos, provocando uma reação imediata e explosiva, com liberação de diversas substâncias químicas, produzindo inflamação, que dará origem aos sintomas da alergia. ${ }^{5}$ Os principais tipos de alergia encontrados em idosos são rinite, asma, dermatite de contato, urticária e hipersensibilidade medicamentosa. ${ }^{2}$

$\mathrm{O}$ objetivo deste estudo foi pesquisar as características epidemiológicas e os aspectos clínicos dos quadros de hipersensibilidade observados na população idosa atendida pela especialidade de geriatria.

\section{MÉTODOS}

Por meio de um delineamento transversal, foram estudados pacientes idosos do Ambulatório de Geriatria da Faculdade de Medicina de Marília (FAMEMA), o qual presta atendimento a pessoas com maior gravidade e/ou complexidade, encaminhados pelos serviços de atenção básica da região de Marília, cidade situada no centro oeste do Estado de São Paulo.

A coleta de dados foi realizada entre julho de 2012 e abril de 2013. Foi utilizada uma entrevista estruturada com perguntas fechadas, apresentadas pelo pesquisador. O questionário continha questões sobre aspectos sociodemográficos e perguntas específicas sobre características clínicas de doenças alérgicas, como alergias respiratórias, alergias de pele, alergias medicamentosas e alimentares, para identificar possíveis manifestações alérgicas no paciente idoso.
As entrevistas foram aplicadas por uma enfermeira e acompanhada por um médico especialista em alergia.

Os critérios de inclusão para esta amostra foram idade igual ou superior a 60 anos e condições de responder à entrevista, ou com acompanhantes capacitados para respondê-la. Os pacientes foram selecionados de acordo com a ordem de chegada ao ambulatório, em sequência, independente do sexo e idade, até atingir um número amostral de 106 pacientes. Este número foi um pouco acima do $\mathrm{N}$ mínimo calculado de 95 pacientes dentro de uma confiabilidade de $95 \%$, um intervalo de confiança de $6 \%$ e uma prevalência de alergia de $10 \%$ em idosos, de acordo com estudo já realizado. ${ }^{6}$ Para o índice de fragilidade, consideraram-se três possibilidades: independente, frágil ou gravemente comprometido. ${ }^{7}$ Para isto foram aplicados os critérios clínicos a seguir relacionados:

1. Independente: pacientes independentes em todas as atividades diárias com curto tempo de doença aguda (p. ex. pneumonia).

2. Frágil (pelo menos um dos seguintes critérios): acidente vascular cerebral, doença crônica incapacitante, confusão mental, dependência das atividades diárias, depressão, quedas, mobilidade comprometida, incontinência fecal ou urinária, desnutrição, polifarmácia, escara de pressão, acamado, restrições motoras, deficiência sensorial, problemas familiares ou socioeconômicos.

3. Gravemente comprometido: doença terminal ou dependência importante das atividades diárias.

Com o intuito de se identificar hipersensibilidade medicamentosa nos pacientes idosos, consideramos como sintomas de alergia medicamentosa aqueles que ocorreram após a administração de um fármaco, independente do tipo de reação apresentada. As reações de hipersensibilidades medicamentosas podem ter diferentes apresentações, como urticária, dermatite e broncoespasmo. Nesta pesquisa foi perguntado ao paciente se o mesmo havia apresentado alguma forma de reação medicamentosa, procurando definir qual medicamento, entretanto sem especificar qual a reação.

Para os dados quantitativos de distribuição normal foi utilizada média como medida de tendência central e desvio padrão como medida de dispersão. As variáveis categóricas foram expressas em números absolutos e percentagens.

A coleta de dados foi realizada após a aprovação do projeto pelo Comitê de Ética em Pesquisa da FAMEMA, 
sob protocolo $\mathrm{n}^{\circ} 206 / 12$, sendo dada aos participantes a garantia da livre adesão, com a assinatura de um Termo de Consentimento Livre e Esclarecido, respeitando-se os procedimentos previstos na Resolução 196/96 do Conselho Nacional de Saúde.

\section{RESULTADOS}

Foram entrevistados 106 pacientes; destes, 17 $(16 \%)$ eram do sexo masculino e $89(84 \%)$ eram do sexo feminino; apresentaram idade entre 60 e 92 anos, média de 74,82 $\pm 8,57$ anos. Quanto ao estado civil, 49 (46\%) eram viúvos, 48 (45\%) casados/com companheiro e nove $(9 \%)$ solteiros. Em relação à escolaridade, 70 $(66 \%)$ realizaram o ensino fundamental, quatro (4\%) o ensino médio, 31 (29\%) não estudaram, e apenas um (1\%) tinha curso superior.

Em relação à ocupação atual dos pacientes, 64 (60\%) eram aposentados, 12 (11\%) eram do lar, um ( $1 \%$ ) atuava em construção civil, e 29 (28\%) exerciam outras atividades. Quanto à profissão pregressa, 36 (34\%) eram do lar, quatro (4\%) eram agricultores, dois $(2 \%)$ eram professores, um $(1 \%)$ trabalhava no comércio, um (1\%) trabalhava em construção civil e $62(58 \%)$ exerciam outras atividades.

Os resultados das principais doenças identificadas nos pacientes entrevistados estão apresentados na Tabela 1. Em relação ao índice de fragilidade dos pacientes entrevistados, $64(60,4 \%)$ idosos foram classificados como frágeis e $44(39,6 \%)$ foram considerados independentes.

Em relação ao uso de medicamentos, apenas três pacientes dos 106 relataram não fazer uso de qualquer medicamento, com um número médio de medicações utilizadas de 5,4 $\pm 3,02$, ficando evidente a presença de polifarmácia nestes pacientes. Os tipos de medicamentos usados estão descritos na Tabela 2.

Dos 106 pacientes entrevistados, $67(63,2 \%)$ relataram manifestações alérgicas, como alergia respiratória (rinite e/ou asma), queixas relativas à pele e respostas relacionadas a possível alergia medicamentosa. Quanto aos sintomas respiratórios, os mais frequentemente identificados como alérgicos foram rinite, isolada ou concomitante com asma, e asma. Outra condição referida como alergia respiratória foi sinusite. Em relação às queixas relativas à pele, identificamos possíveis manifestações alérgicas, como prurido e dermatite de contato. Entre os pacientes que relataram prurido, a maior parte apresentou pele seca, segundo avaliação do entrevistador. A hipersensibilidade de origem possi- velmente medicamentosa esteve presente em 14 $(13,2 \%)$ pacientes. Estes resultados estão descritos na Tabela 3.

Tabela 1. Prevalências das doenças identificadas em106 pacientes idosos entrevistados no Ambulatório de Geriatria da Faculdade de Medicina de Marília.

\begin{tabular}{|c|c|}
\hline Doenças & N (\%) \\
\hline Cardiovasculares & $75(70,7)$ \\
\hline Reumatológicas & $67(63,3)$ \\
\hline Diabetes & $27(25,4)$ \\
\hline Demência & $21(19,8)$ \\
\hline Distúrbios da Tireoide & $19(17,9)$ \\
\hline Depressão & $7(6,6)$ \\
\hline Doença pulmonar obstrutiva crônica & $2(2,8)$ \\
\hline Outras doenças & $21(19,8)$ \\
\hline
\end{tabular}

A soma das porcentagens ultrapassa os $100 \%$ porque muitos pacientes apresentavam mais de uma doença.

Tabela 2. Porcentagens dos medicamentos utilizados em106 pacientes idosos entrevistados no Ambulatório de Geriatria da Faculdade de Medicina de Marília.

\begin{tabular}{|lc|}
\hline \multicolumn{1}{c}{ Medicamentos } & N (\%) \\
\hline Cardiovasculares & $82(77,3)$ \\
\hline Reumatismo/osteoporose & $49(46,2)$ \\
\hline Antidepressivo & $44(41,5)$ \\
\hline Estômago & $30(28,3)$ \\
\hline Distúrbios da Tireoide & $29(27,3)$ \\
\hline Diabetes & $26(24,5)$ \\
\hline Anti-inflamatórios não hormonais & $26(24,5)$ \\
\hline Demência & $8(7,5)$ \\
\hline Alergias & $2(1,8)$ \\
\hline Outros & $39(36,7)$ \\
\hline
\end{tabular}

A soma das porcentagens ultrapassa os $100 \%$ porque muitos pacientes utilizavam mais de um medicamento.

Tabela 3. Grupos de manifestações identificadas como alérgicas e tipos de manifestações respiratórias e cutâneas em106 pacientes idosos entrevistados no Ambulatório de Geriatria da Faculdade de Medicina de Marília.

\begin{tabular}{lc}
\multicolumn{1}{c}{ Tipo de manifestação } & N (\%) \\
\hline Manifestações alérgicas & $67(63,2)$ \\
Respiratórias & $56(52,8)$ \\
Cutâneas & $47(44,3)$ \\
Medicamentosas & $14(13,2)$ \\
Manifestações alérgicas respiratórias & $56(52,8)$ \\
Rinite & $42(75)$ \\
Asma & $8(14)$ \\
Asma e rinite & $5(9)$ \\
Outras & $1(2)$ \\
Manifestações cutâneas & $47(44,3)$ \\
Prurido & $44(93,6)$ \\
Dermatite de contato & $3(6,4)$ \\
\hline
\end{tabular}


Os principais medicamentos responsabilizados por reações alérgicas pelos pacientes foram os antibióticos $(42,8 \%)$ e os anti-inflamatórios não esteroides $(28,6 \%)$, enquanto os outros $28,6 \%$ foram atribuídos a outros tipos de medicamentos.

Entre os 67 pacientes que referiam doenças alérgicas, apenas oito (12\%) faziam uso de medicamentos antialérgicos. Destes, dois faziam uso de anti-histamínico, dois de corticoide nasal, três da associação broncodilatador mais corticoide inalatório, e um de soro fisiológico nasal.

\section{DISCUSSÃO}

Com relação à prevalência das alergias identificadas nos pacientes entrevistados no ambulatório de geriatria, dos 106 entrevistados, 67 (63,2\%) apresentavam alguma manifestação possivelmente alérgica, o que representa uma quantidade considerável, sendo que estes distúrbios, apesar de não se apresentarem espontaneamente como causas motivadoras das consultas, podem interferir de forma importante na qualidade de vida dessas pessoas.

Um interessante estudo epidemiológico de manifestações alérgicas em idosos realizado na Itália, em uma unidade de alergologia, ao longo de um período de três meses no início de 2008 , mostrou que $15 \%$ pertenciam à população idosa e entre estes $51,8 \%$ estavam sofrendo de uma reação alérgica a drogas. Manifestações cutâneas, incluindo urticária e eczema, foram responsáveis por $71,4 \%$ dos casos, rinite estava presente em $16,8 \%$ dos pacientes e alergia alimentar em $8 \%{ }^{4}$

No presente estudo, os sintomas de alergias respiratórias apresentaram maior prevalência. Das manifestações respiratórias, a que apresentou maior prevalência foi a rinite. Segundo Miyake, ${ }^{8}$ no idoso, a irritação e secura nasal podem provocar piora ou prolongamento de doenças como rinite alérgica, rinite irritativa e rinossinusites. A rinite não alérgica no idoso, ocorrida pela disfunção do sistema nervoso autônomo com predomínio parassimpático, é caracterizada por coriza e obstrução nasal que pode piorar durante as refeições, por alimentos quentes e temperados ou durante o decúbito.

Apesar de que a asma no idoso apresenta uma prevalência semelhante à de outras faixas etárias, o diagnótico muitas vezes não é evidênciado devido à idéia de que essas doenças sejam pouco frequentes nessa população. Várias doenças podem ser confundidas com a asma, e no idoso é comum a mesma ser confundida com doença pulmonar obstrutiva crônica, principalmente em pacientes fumantes, sendo que o tabagismo é um fator de risco para morbidade e mortalidade por asma. ${ }^{9}$ Além disso, no idoso, a função pulmonar diminuída e a redução da imunidade, que predispõe a infecções respiratórias, são fatores condicionantes para a exacerbação da asma, o que pode causar maior probabilidade de internações. ${ }^{10}$

Existem poucos estudos a respeito de características clínicas de idosos asmáticos. Em estudo realizado com idosos acima de 65 com asma, Rodrigues et al. ${ }^{11}$ concluiram que pacientes que apresentavam a doença por um período maior relatavam menos queixas das mesmas. Esses autores citam ainda outro estudo que relata que pacientes idosos têm a percepção da dispneia prejudicada. Analisando a situação, concluise que existem várias características que prejudicam o diagnóstico de asma no indivíduo idoso. Sendo assim, talvez a prevalência da doença nessa faixa etária seja maior do que a apresentada na literatura científica. ${ }^{11}$

As manifestações cutâneas sugestivas de alergias também foram um dos principais aspectos identificados nos pacientes entrevistados, sendo o prurido a manifestação mais prevalente. O prurido é uma manifestação muito comum na população idosa, com ou sem exantema, sendo a pele seca a principal causa deste sintoma. Entretanto, é necessário observar se as causas do prurido não são de outra origem, como medicamentosa, psicogênica, doenças sistêmicas, etc. A xerose (pele seca) também é bastante comum em indivíduos acima de 60 anos, principalmente em regiões como as pernas, por causa da diminuição da atividade das glândulas sebáceas e sudoríparas nessa faixa etária.

Ribeiro et al. ${ }^{12}$ realizaram um estudo no ambulatório geral do Serviço de Dermatologia do Hospital do Servidor Público Estadual de São Paulo, em 2006, sobre prevalência de dermatoses em pacientes da quarta idade (acima dos 80 anos). Foram examinados 210 pacientes que tiveram a média de idade de 84 anos. Destes, 10 apresentaram prurido, 10 apresentaram xerose e quatro pacientes apresentaram dermatite de contato, entre um total de 12 dermatoses identificadas, sendo a mais prevalente a ceratose actínica (distúrbio não alérgico), presente em 48 pacientes. Os autores também relatam que a xerose é uma característica da pele do idoso e que pode ter influência genética. É mais comum em regiões do tronco, membros superiores e inferiores, e é uma das principais causas do prurido. ${ }^{12}$

A prevalência de hipersensibilidade a drogas é de 7\% na população em gera ${ }^{13} \mathrm{e}$ essas reações representam $15 \%$ das reações adversas medicamentosas, sendo os principais medicamentos causadores das mesmas os antibióticos e os anti-inflamatórios não hormonais. Embora a frequência de queixas referindo reações a drogas tenha sido maior no presente estudo, as principais causas, 
como sendo os antibióticos e os anti-inflamatórios não hormonais, foram igualmente referidas pelos idosos entrevistados. De acordo com Bernd, ${ }^{14}$ reações alérgicas e pseudo-alérgicas representam cerca de $25 \%$ das reações adversas medicamentosas e são muito frequentes principalmente nos ambulatórios de alergia e clinica.

Dentre todos os pacientes tratados com medicamentos, entre $2 \%$ a $6 \%$ apresentam reações de hipersensibilidade medicamentosa, sendo $95 \%$ dos casos erupções cutâneas não urticariformes e 5\% urticária. Os principais medicamentos que provocam essas reações são também os antimicrobianos e os anti-inflamatórios não hormonais. ${ }^{15}$ Campos-Fernández et al. realizaram um estudo coorte em uma unidade de terapia intensiva durante um período de oito meses, avaliando reações cutâneas adversas medicamentosas. Mostraram que os principais fatores de risco para estas reações foram sexo feminino, obesidade, idade superior a 60 anos e alguma disfunção do sistema imune. Foram internados 190 pacientes durante o período do estudo, sendo que $22(11,6 \%)$ apresentaram reação cutânea adversa medicamentosa, dos quais $45 \%$ eram pacientes com idade superior a 60 anos. ${ }^{15}$

Verificamos neste estudo que os idosos tiveram uma significativa prevalência de manifestações possivelmente alérgicas, o que pode interferir em sua qualidade de vida. Os principais quadros identificados foram alergias respiratórias com destaque para a rinite, tendo esta, muitas vezes, características não alérgicas, predominando a obstrução nasal e a coriza em relação ao prurido e aos espirros. Também se sobressaíram as manifestações cutâneas, com ênfase para a queixa do prurido cutâneo associado à xerose, além da hipersensibilidade medicamentosa, principalmente aos anti-inflamatórios não hormonais e antibióticos.

Concluindo, apesar de termos encontrado muitas vezes manifestações não propriamente alérgicas, como no caso do prurido, o qual se correlacionou principalmente com a pele seca, e da rinite, com o componente mais obstrutivo do que pruriginoso, caracterizando mais especificamente a rinite não alérgica, tais manifestações podem não ser adequadamente valorizadas na abordagem do idoso dentro das diferentes especialidades e, por conseguinte, trazer implicações negativas na qualidade de vida do mesmo. Acreditamos que são manifestações frequentes e que, portanto, devemos sempre estar atentos a elas. Também, pelos poucos trabalhos sobre o assunto, e devido ao crescimento exponencial desta faixa etária no Brasil, são necessários mais estudos direcionados a esta área.

\section{REFERÊNCIAS}

1. Andrade MA, Silva MVS, Freitas O. Assistência farmacêutica como estratégia para o uso racional de medicamentos em idosos. Semina Cienc Biol Saúde. 2004;25(1):55-63.

2. Cardona V, Guilarte M, Luengo O, Labrador-Horrillo M, Sala-Cunill A, Garriga T. Allergic diseases in the elderly. Clin Transl Allergy. 2011;1(1):e11.

3. Becerril-Ángeles M, Vázquez-Merino CL, Ángeles-Garay U, Alvarado-Moctezuma LE, Vilchis-Guízar E. Prevalencia de enfermedades alérgicas en adultos mayores. Rev Alerg Mex. 2008;55(3):85-91.

4. Ventura MT, D'Amato A, Giannini M, Carretta A, Tummolo RA, Buquicchio R. Incidence of allergic diseases in an elderly population. Immunopharmacol Immunotoxicol. 2010;32(1):e165-70.

5. Pecher AS. Asma brônquica no idoso. Rev Para Med. 2007;21(3):1-5.

7. Carvalho SMR, Castro AA. Projeto de pesquisa (parte VIII - método estatístico/tamanho da amostra). In: Castro AA. Planejamento da pesquisa [Internet]. São Paulo: 2001. p. 1-9 [cited 2012 Feb 23]. Available from: http://www.decisaoclinica.com/planejamento/pdf/ lv4_10_tamanho.pdf

8. Winograd CH, Gerety MB, Chung M, Goldstein MK, Dominguez F Jr, Vallone R. Screening for frailty: criteria and predictors of outcomes. J Am Geriatr Soc. 1991;39(8):778-84.

9. Miyake MAM. Afecções otorrinolaringológicas no idoso: o impacto da "polifarmácia”. Rev Hosp Univ Pedro Ernesto. 2012;11(3):83-91.

10. Souza LNS, Volquind GG, Lazzari CA. Tratamento de asma no idoso atendido em uma Unidade Básica de Saúde em Porto Alegre. Mom Perspectiv Saúde. 2005;18(2):29-34.

11. Rodrigues PCO, Ignotti E, Rosa AM, Hacon SS. Distribuição espacial das internações por asma em idosos na Amazônia Brasileira. Rev Bras Epidemiol. 2010;13(3):523-32.

12. Ribeiro TEL, Silva ECF, Meneses SLS, Lopes AJ. Correlação dos achados clínicos com os parâmetros funcionais em idosos portadores de asma. Rev Port Pneumol. 2009;15(6):1029-41.

13. Sittart JAS, Zanardi FHT. Prevalência das dermatoses em pacientes da $4^{\mathrm{a}}$ idade. Rev Soc Bras Clin Med. 2008;6(4):125-9.

14. Ensina LF, Fernandes FR, Di Gesu G, Malaman MF, Chavaria ML, Bernd LAG. Reações de hipersensibilidade a medicamentos. Rev Bras Alergia Imunopatol. 2009;32(2):42-7.

15. Bernd LAG. Alergia a medicamentos. Rev Bras Alergia Imunopatol. 2005;28(3):125-32.

16. Campos-Fernández M del M, Ponce-De-León-Rosales S, Archer-Dubon C, Orozco-Topete R. Incidence and risk factors for cutaneous adverse drug reactions in an intensive care unit. Rev Invest Clin. 2005;57(6):770-4. C 